\title{
Tracheobronchial Calcification with Sinus Destruction
}

Sah RB ${ }^{1}$, Gothi $\mathbf{D}^{1^{*}}$, Singhal $\mathbf{A}^{2}$, Jain $\mathbf{S}^{3}$ and Gupta $\mathbf{R}^{1}$

${ }^{1}$ Department of Pulmonary Medicine, ESI- PGIMSR, Delhi, India

${ }^{2}$ Department of Radiology, ESI-PGIMSR, Delhi, India

${ }^{3}$ Senior Consultant Pathologist, Onquest Laboratories, India

*Corresponding author: Gothi D, Department of Pulmonary Medicine, ESI- PGIMSR, Delhi, India, Tel: +919810080751; Fax: +919810080751; E-mail: diptigothi@gmail.com

Received date: November 16, 2016; Accepted date: November 26, 2016; Published date: November 28, 2016

Copyright: (C) 2016 Sah RB, et al. This is an open-access article distributed under the terms of the Creative Commons Attribution License, which permits unrestricted use, distribution, and reproduction in any medium, provided the original author and source are credited.

\begin{abstract}
A 34-year-old lady presented with history suggestive of bronchial asthma \& allergic rhinosinusitis. Computerized tomogram (CT) of thorax showed nodular calcification of trachea with sparing of the posterior wall. CT paranasal sinus revealed sinusitis with bone destruction. Bronchoscopy and bronchial biopsy was consistent with the diagnosis of tracheobronchopathia osteochondroplastica (TPO). Diagnosis of atrophic rhino sinusitis was established based on the diagnostic criteria. Sinobronchial symptoms have been described in TPO, but destruction of sinus due to atrophic rhinosinusitis has not been reported.
\end{abstract}

Keywords: Tracheobronchopathia osteochondroplastica (TPO); Atrophic rhinosinusitis; Tracheobronchial calcification

\section{Introduction}

Many diseases can lead to tracheobronchial calcification. Clinicoradiological correlation can identify the etiology of tracheobronchial calcification in most cases.

Tracheobronchopathia Osteochondroplastica (TPO) is one of the rare causes of tracheobronchial calcification. Sinobronchial involvement due to TPO has been documented in past, however CT sinus findings in cases of TPO have not been described earlier.

\section{Case Report}

A 34 year old non-smoker, homemaker was referred for the management of bronchial asthma. She complained of moderate-severe rhinitis, sinusitis, progressively increasing dyspnea on exertion and cough for 7 years. She denied history of paroxysmal breathlessness, emergency hospital visits or use of inhaled bronchodilators.

There was no history of recurrent lung infections in childhood. She had a history of atopy in self. Her paternal uncle had a history of asthma. She also had recurrent purulent nasal discharge and anosmia for the last two years. She did not have any systemic complaints and there was no past history of sinus surgery.

At presentation her vitals were normal. General physical examination was unremarkable. Examine of para-nasal sinuses (bilateral maxillary and frontal) showed tenderness. There was nasal crusting and patulous nasal passage on nasal examination.

Examination of ear was normal. Respiratory system examination showed bilaterally equal breath sound with polyphonic rhonchi auscultated all over the chest. Other system examination was normal.

Routine microscopic examination of her urine sample was normal. She had a chest radiograph done 2 weeks ago from elsewhere, showed bilateral mid-zone non-homogenous opacities (Figure 1a). These opacities had reduced at presentation and had subsided after 1 month (Figure 1b).

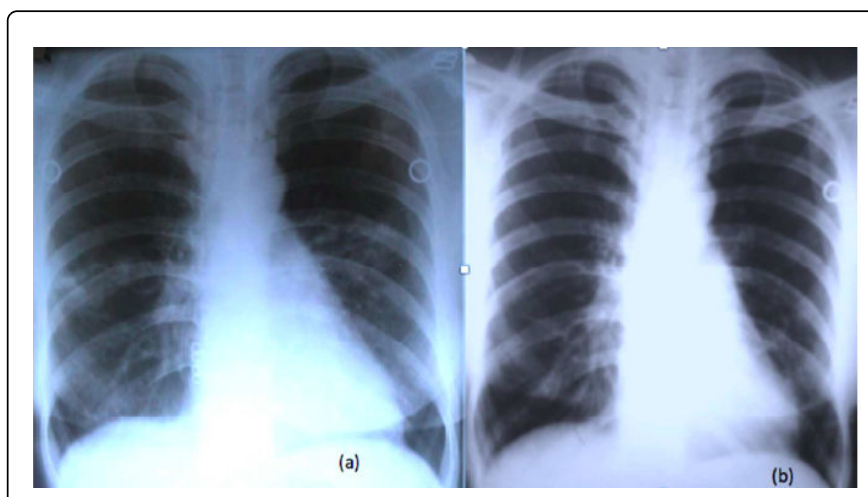

Figure 1: 1a- Chest radiograph done 2 week ago from outside showing bilateral mid-zonal non-homogenous opacities. 1b- Chest radiograph after 1 month shows resolution of lesion.

Her routine investigations showed hemoglobin $11.8 \mathrm{gm} / \mathrm{dl}$, total leucocyte count of $10400 / \mathrm{mm}^{3}$ with differential counts of polymorphs $67 \%$, lymphocytes $29 \%$ and eosinophil $4 \%$. Her biochemical test results were normal. Total immunoglobulin (Ig) E level was $600 \mathrm{IU} / \mathrm{ml}$.

Spirometry showed obstructive defect i.e. ratio of forced expiratory volume in the first second to forced vital capacity of $61 \%$ and forced expiratory volume in the first second of 1.681 (59\% predicted) with significant bronchodilator reversibility of $390 \mathrm{ml}$ and $17 \%$. Serology for aspergillus showed anti-aspergillus IgG level of $7.8 \mathrm{IU} / \mathrm{ml}($ normal $<8$ ), anti-aspergillus IgE level of $0.22 \mathrm{IU} / \mathrm{ml}$ (normal<0.35) and antiaspergillus IgM level of $4.20 \mathrm{IU} / \mathrm{ml}$ (normal<8.00).

Computerised tomogram (CT) of thorax revealed diffuse thickening of walls of trachea, main and segmental bronchi along with submucosal nodular calcifications causing irregular luminal narrowing. Posterior wall of trachea was spared (Figure 2). 
Citation: Sah RB, Gothi D, Singhal A, Jain S, Gupta R (2016) Tracheobronchial Calcification with Sinus Destruction. J Clin Respir Dis Care 2:

Page 2 of 4

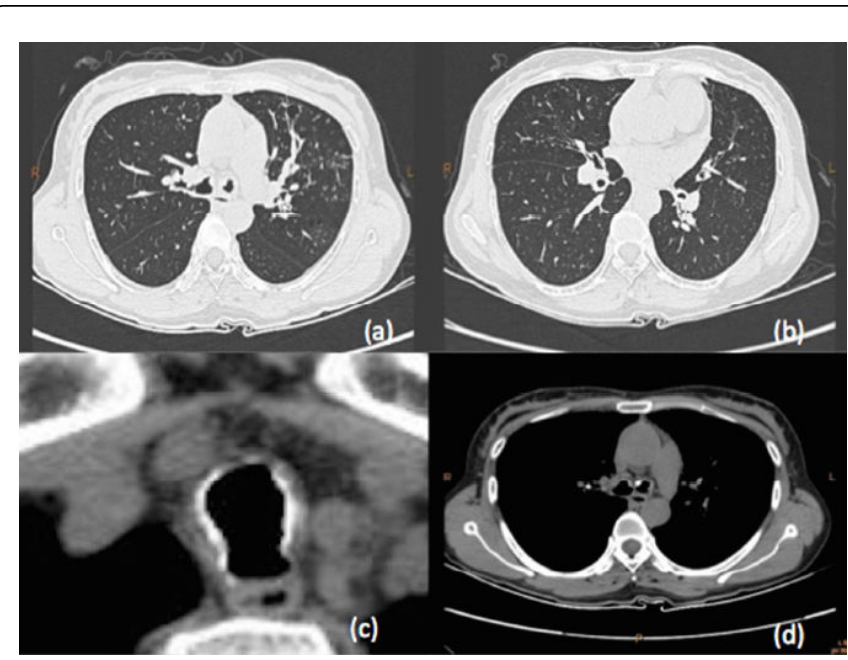

Figure 2: $2 \mathrm{a}$ and $2 \mathrm{~b}$ - CT of thorax- lung window coronal section at the level of tracheal bifurcation and transverse fissure showing thickening of bronchial walls along with nodular calcification and luminal narrowing. $2 \mathrm{c}$ and $2 \mathrm{~d}-\mathrm{CT}$ of thorax mediastinal window coronal section at the level of trachea and carina shows thickening of tracheal and bronchial walls along with nodular calcification and luminal narrowing and sparing of posterior wall.

Peripheral subsegmental areas of atelectasis and consolidation were also seen in bilateral upper lobes. Tomogram of para-nasal sinuses (PNS) showed pansinusitis with erosive destruction of walls of sinuses and turbinates, which appeared atrophic (Figure 3). Cytoplasmic and peripheral antineutrophil antibodies were 2.19 (normal<7 IU/ml) and $3.22($ normal $<7 \mathrm{IU} / \mathrm{ml})$ respectively.

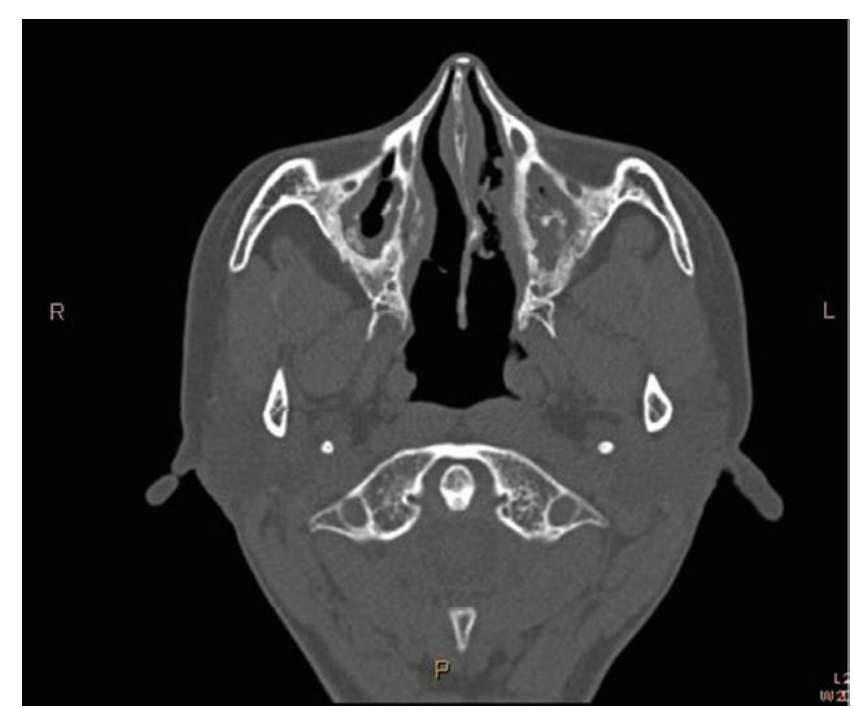

Figure 3: Computerized tomogram of paranasal sinuses showing pansinusitis with bone destruction.

She was further advised positron emission tomography (PET) scan which showed metabolically inactive irregular mural thickening of anterior and lateral wall of trachea, bilateral main and segmental bronchi with multiple calcified nodules predominantly in left upper, lower lobe and right middle bronchi (Figure 4 ). There was no activity in paranasal sinuses.

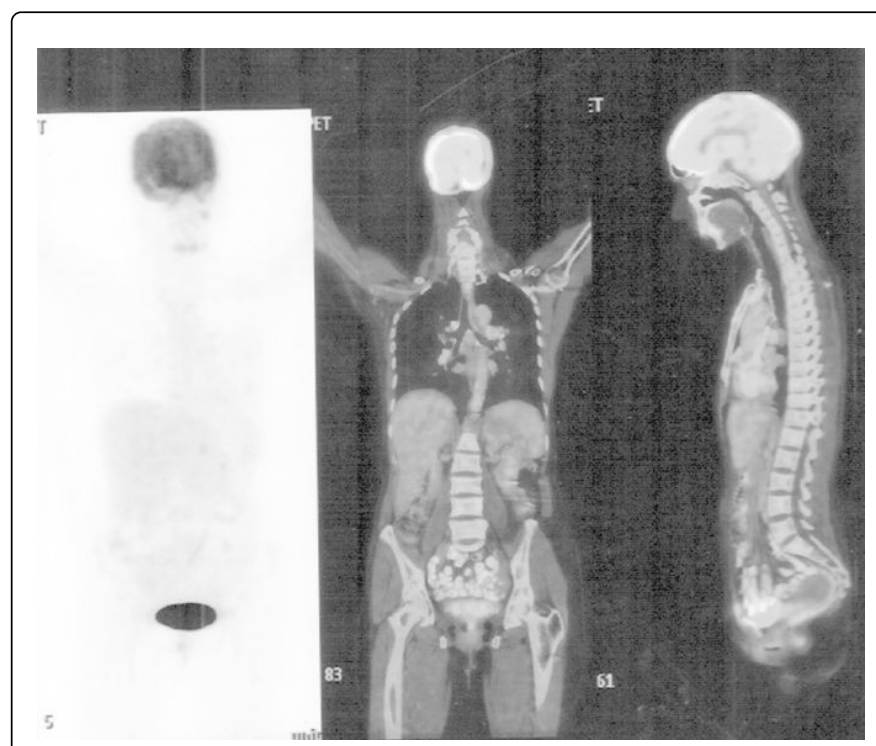

Figure 4: PET scan showing- metabolically inactive lesions.

Bronchoscopy revealed multiple white to yellowish hard nodules with cobblestone appearance projecting into the lumen of tracheobronchial tree. The nodules were observed all along the tracheal lumen, main bronchi, segmental bronchi and subsegmental bronchi with sparing of subglottic region and posterior wall of trachea (Figure 5). The trachea and bronchi were narrowed. The subsegmental bronchi could not be negotiated due to narrowing.

Histopathological examination of biopsy showed spicules of lamellar bone, fat cells and bits of metaplastic squamous epithelium (Figure 6). She was treated with long acting beta agonists, inhaled steroids and steroidal nasal spray because of atopic symptoms and good bronchodilator reversibility. Atrophic rhinosinusitis was managed conservatively with nasal irrigation with normal saline. The patient is taking prescribed medication regularly and has reported significant improvement in symptoms. She is on regular follow up for possible requirement of intervention for tracheobronchial nodule and atrophic rhinosinusitis.

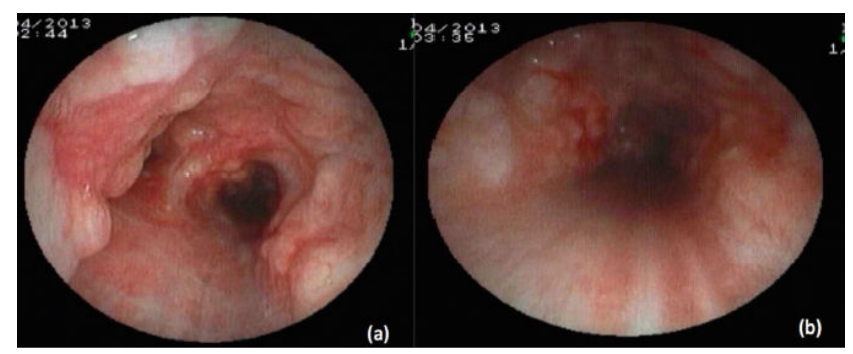

Figure 5: 5a and 5b- Bronchoscopy image showing- multiple, nodules in the trachea and main bronchi with characteristic 'cobblestone' appearance and sparing of the posterior walls. 


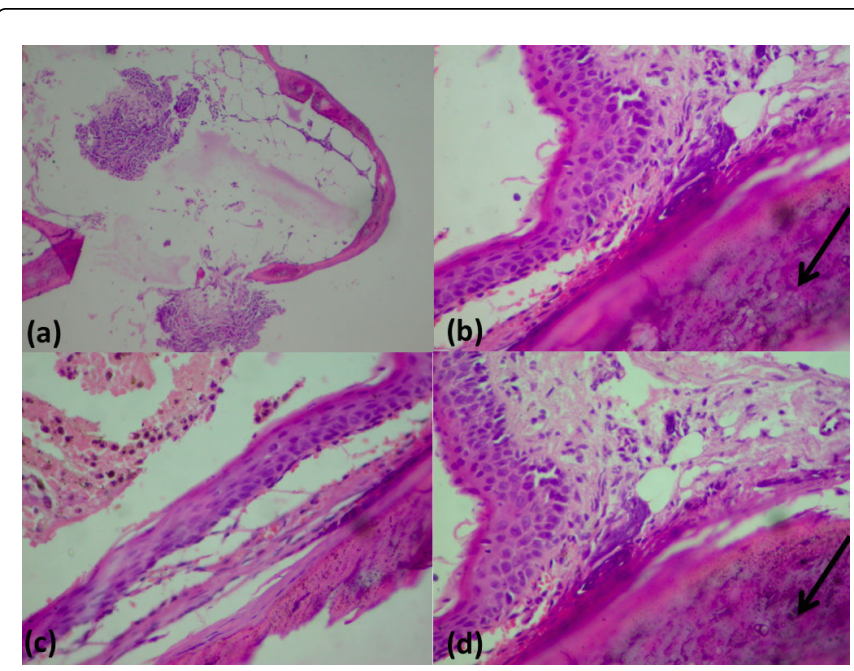

Figure 6: 6a- Low power showing spicules of bone, fat cells and bits of metaplastic squamous epithelium. $6 \mathrm{~b}$ and $6 \mathrm{~d}-\mathrm{High}$ magnification shows bone (black arrow) below the squamous epithelium. Scanty cartilage cells also seen. $6 c$-Medium power shows similar features.

\section{Discussion}

Tracheobronchopathia Osteochondroplastica (TPO) is an idiopathic, benign disorder of large airways. Till now approximately 400 cases have been reported in literature [1]. It occurs more frequently in males over 50 years, although cases have been reported in younger individuals and females. The severity of symptoms in TPO depends on the extent of airway narrowing arising due to submucosal nodules projecting in the airway lumen. TPO is a slowly progressive disorder, remains stable for many years. Majority of TPO patients are asymptomatic until the tracheal diameter falls below $1 \mathrm{~cm}$. Others have cough, breathlessness, wheezing, hemoptysis and recurrent respiratory infections. Often they are diagnosed as asthma or chronic bronchitis. Our patient too was being referred for the management of asthma. But, atypical presentation i.e. absence of paroxysmal dyspnea and presence of abnormal chest radiograph led to further investigation. Pulmonary function test may be normal or show an obstructive pattern as seen in our case [2].

She was diagnosed to have atrophic rhinosinusitis as per diagnostic criteria suggested by Ly TH et al. [3]. These criteria are:

- Patient-reported recurrent epistaxis or episodic anosmia;

- Physician-documented nasal purulence, nasal crusting, chronic inflammatory disease of the upper airway

- Two or more sinus surgeries

If a patient with chronic rhinosinusitis demonstrates two or more clinical features for 6 months and longer it indicates atrophic rhinosinusitis [3]. She satisfied two of the criteria i.e. episodic anosmia and nasal crusting. Large roomy nasal cavity on nasal examination corroborated with the diagnosis of atrophic rhinosinusitis.

Chest radiograph in TPO is usually normal, but there can be areas of atelectasis and ill-defined opacities [4] as in our case. The CT thorax in our patient showed nodular calcification of tracheobronchial tree with sparing of the subglottic trachea and the posterior wall.
Differential diagnosis of tracheobronchial calcification aretracheomalacia, saber sheath trachea, Mounier-Kuhn syndrome, Wegener's granulomatosis, endobronchial sarcoidosis, inflammatory bowel disease, papillomatosis, amyloidosis, relapsing polychondritis, and TPO [5]. Tracheomalacia has lunate-shaped trachea, saber sheath trachea has narrowed coronal diameter whereas, Mounier-Kuhn has increased tracheobronchial diameter. The tracheal anatomy apart from narrowing was normal in our case. Wegener's granulomatosis almost always affect upper airways and subglottic trachea [6]. Kidneys are involved in $80 \%$ cases. Elevation of cytoplasmic antineutrophil antibodies is common. Moreover, there are metabolically active areas of inflammation on PET scan in Wegener's granulomatosis. In our case the lesions were metabolically inactive. Sarcoidosis and inflammatory bowel disease have systemic symptoms, which were absent in our case. Papillomatosis and amyloidosis tends to involve the airway concentrically, as opposed to TPO, which spares the posterior wall. The only other disease apart from TPO, which spare the posterior wall, is relapsing polychondritis. In relapsing polychondritis however besides tracheal calcification there is recurrent chondritis of ear, nose and respiratory tract along with inflammatory non-erosive polyarthritis and ocular inflammation. Also, in relapsing polychondritis there is high attenuation thickening of tracheal and bronchial walls along with destruction of tracheal cartilaginous rings, whereas presence of focal, coarse calcification/ossification favors TPO [7]. The clinicoradiological findings, thus suggested the diagnosis of TPO.

Though, CT chest and clinical correlation was highly suggestive of TPO, sinus destruction on CT PNS could not be explained by TPO. The sinus destruction corroborated with the clinical diagnosis of atrophic rhinosinusitis [8]. A few case reports of TPO with sinobronchial symptoms have been described [9]. Occurrence of atrophic rhinitis has been observed in a few case series as well $[1,10]$. It has also been suggested that the presence of cough, dyspnea, hemoptysis, or recurrent sinobronchial infections in a patient with atrophic rhinitis should raise the suspicion of TPO [11]. But TPO with atrophic rhinosinusitis has not been described so far. It is possible that if previous cases of atrophic rhinitis and sinobronchial symptoms had been studied in detail, diagnosis of atrophic rhinosinusitis could have been established. There is a possibility that atrophic rhinosinusitis is secondary to TPO, as chronic inflammatory conditions are known to be associated with atrophic rhinosinusitis. More cases of TPO are required to be studied in detail with CT PNS to establish that rhinosinusitis in TPO is secondary.

The diagnosis of TPO is made on bronchoscopy. The classical findings are sessile cartilaginous and/or bony nodules with normal overlying mucosa sparing the posterior wall, producing a beaded appearance. This appearance is also called rock garden or cobblestone appearance of tracheobronchial tree 8 as seen in our case. Biopsy of these nodules is essential to confirm the diagnosis. The biopsy confirmed the diagnosis of TPO in our case. Treatment of TPO is necessary for symptomatic cases only, as no definitive management is available and the prognosis is generally good. Conservative management with antibiotics is required for those with recurrent respiratory tract infections, while others who develop obstructive symptoms may require interventions like removal of nodules by forceps, laser photoevaporation or cryotherapy or external beam irradiation or endobronchial stent placement or linear tracheoplasty $[1,12]$. 
Citation: Sah RB, Gothi D, Singhal A, Jain S, Gupta R (2016) Tracheobronchial Calcification with Sinus Destruction. J Clin Respir Dis Care 2: 123. doi:10.4172/2472-1247.1000123

Page 4 of 4

\section{References}

1. Jabbardarjani HR, Radpey B, Kharabian S, Masjedi MR (2008) Tracheobronchopathia osteochondroplastica: presentation of ten cases and review of the literature. Lung 186: 293-297.

2. Lundgren R, Stjernberg NL (1981) Tracheobronchopathia osteochondroplastica: a clinical bronchoscopic and spirometric study. Chest 80: 706-709.

3. Ly TH, deShazo RD, Olivier J, Stringer SP, Daley W, et al. (2009) Diagnostic criteria for atrophic rhinosinusitis. Am J Med 122: 747-753.

4. Prakash UB, McCullough AE, Edell ES, Nienhuis DM (1989) Tracheopathia osteoplastica: familial occurrence. Mayo Clin Proc 64: 1091-1096.

5. Chung JH, Kanne JP, Gilman MD (2011) CT of diffuse tracheal diseases. AJR Am J Roentgenol 196: W240-W246.

6. Prince JS, Duhamel DR, Levin DL, Harrell JH, Friedman PJ (2002) Nonneoplastic lesions of the tracheobronchial wall: radiologic findings with bronchoscopic correlation. RadioGraphics 22: S215-S230.
7. Kang EY (2011) Large airway diseases. J Thorac Imaging 26: 249-262.

8. DeShazo RD, Stringer SP (2011) Atrophic rhinosinusitis: progress toward explanation of an unsolved medical mystery. Curr Opin Allergy Clin Immunol 11: 1-7.

9. Jindal S, Nath A, Neyaz Z, Jaiswal S (2013) Tracheobronchopathia Osteochondroplastica-A rare or an overlooked entity. Radiology Case 7: 16-25.

10. Härmä RA, Suurkari S (1977) Tracheopathia chondro-osteoplastica: a clinical study of thirty cases. Tracheobronchopathia osteochondroplastica- a rare or an overlooked entity? Acta Otolaryngol. 84: 118-123.

11. Lazor R, Cordier JF (2004) Tracheobronchopathica osteochondroplastica: orphanet encyclopedia.

12. Chroneou A, Zias N, Gonzalez AV, Beamis JF (2008) Tracheobronchopathia Osteochondroplastica. An underrecognized entity. Monaldi Arch Chest Dis 69: 65-69. 